\title{
Energiewende - wofür würde Rudolf Virchow stimmen?
}

\section{Claudio Knüslia, Martin Walter ${ }^{b}$, Jean-Jacques Fasnacht ${ }^{c}$}

${ }^{a}$ Dr. med., Vorstandsmitglied PSR/IPPNW Schweiz, Mitglied FMH, Basel; ' Dr. med., Vorstandsmitglied PSR/IPPNW Schweiz, Mitglied FMH, Grenchen;

${ }^{c}$ Dr. med., Präsident PSR/IPPNW Schweiz, Mitglied FMH, Marthalen

«Soll die Medicin daher ihre grosse Aufgabe wirklich erfüllen, so muss sie in das grosse politische Leben eingreifen; sie muss die Hemmnisse angeben, welche der normalen Erfüllung der Lebensvorgänge im Wege stehen, und ihre Beseitigung erwirken.»

Rudolf Virchow [1]

Rudolf Virchow (1821-1902) - welchem Arzt ist der hochangesehene Begründer der modernen Pathologie nicht vertraut? Am supraklavikulär gelegenen Virchow-Lymphknoten, meist Ausdruck eines metastasierenden Malignoms, kommt auch heute noch kaum ein Medizinstudent vorbei.

Weniger bekannt ist, dass Virchow nicht nur Vertreter der streng naturwissenschaftlich orientierten Heilkunde, sondern auch engagierter liberaler Politiker für eine sozial orientierte Medizin war. Er trat dafür ein, dass Ärzte Volkskrankheiten nicht nur erkennen, sondern diese auch Staatsmännern zur Beseitigung anzeigen sollten [2].

Und geradezu visionär und auch heute modern ist Virchows Mahnung, der kranke Mensch sei als Ganzes aufzufassen [3].

Virchow - konfrontiert mit den damaligen Volkskrankheiten Cholera und Typhus - beriet als Hygieniker Regierungen und trat konsequent dafür ein, dass Berlin um 1870 eine Kanalisation und eine zentrale Trinkwasserversorgung erhielt. Ebenfalls auf Virchow geht die Einrichtung der ersten kommunalen Krankenhäuser in Berlin zurück. Diese Beispiele zeigen, dass für Virchow Medizin und politisches Engagement zusammengehörten, wo es um die zielgerichtete Veränderung schädlicher Umweltverhältnisse zugunsten der Volksgesundheit ging.

\section{Klimawandel ruft nach Energiewende}

Unbestreitbar besteht heutzutage die grösste weltweite medizinische Herausforderung im durch den Menschen verursachten Klimawandel. Saubere Luft, sichere Trinkwasserversorgung, genügend Nahrungsmittel und ein Dach über dem Kopf sind jetzt und in Zukunft elementare Ressourcen, die Gesundheit erst

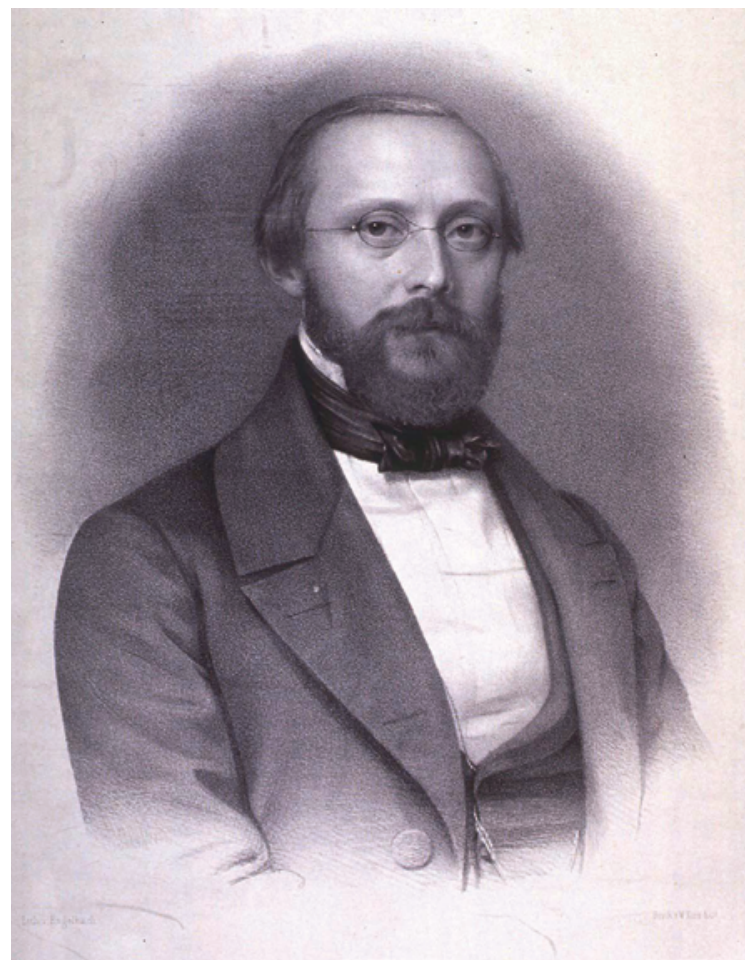

Rudolf Virchow (1821-1902), Begründer der modernen Pathologie, setzte sich für eine sozial orientierte Medizin ein.

ermöglichen. Sie alle sind durch den Klimawandel bedroht. Gemäss WHO ist damit zu rechnen, dass in den nächsten Jahrzehnten jährlich Hunderttausende Menschen an den Folgen des Klimawandels sterben werden [4]. Die direkten medizinischen Kosten werden

Unbestreitbar besteht heutzutage die grösste weltweite medizinische Herausforderung im durch den Menschen verursachten Klimawandel.

jährlich 2-4 Milliarden Dollar betragen. Hauptbetroffene sind bereits jetzt Menschen in Entwicklungsländern. Auch in unseren Breitengraden sind die Folgen für den Einzelnen mehr und mehr spürbar - besonders für Kleinkinder und ältere Menschen. Die Übergänge sind fliessend, von Befindlichkeitsstörungen bis zu lebensbedrohlichen Krankheitszuständen, sei es durch Atemwegspathologien, Allergien, Hitzeschäden oder 
Veränderungen des Spektrums von Infektionskrankheiten.

Die Reduktion der Emissionen, insbesondere von $\mathrm{CO}_{2}$ und anderen Treibhausgasen im Heizungs-, Verkehrs-, Transport- und Nahrungsmittelsektor, wird ausschlaggebend sein. Der steigende Energiekonsum fordert neue Lösungen. Gesundheitliche - ebenso wie ökologische und wirtschaftliche - Argumente verlangen eine

\section{Die Kosten der Energiestrategie2050 sind} im Verhältnis zu deren Nutzen mehr als nur moderat.

zukunftsfähige Energiestrategie. Die anhaltenden Folgen der Katastrophen von Tschernobyl und Fukushima sowie die ungelöste Entsorgung der nuklearen Abfälle rufen dringend nach neuen Energieformen.

\section{Energiestrategie2050 - aus medizinischer Sicht zu bejahen}

Von Virchow haben wir gelernt: Ärzte sollen sich im Dienste einer ganzheitlichen, sozial engagierten Medizin auch in der Öffentlichkeit äussern und einsetzen. Das heisst, dass auch gesundheitliche Aspekte in der laufenden gesellschaftlichen Diskussion Gewicht erhalten müssen [5]. Welche Energiepolitik auch immer betrieben wird: Eine nachhaltige Verbesserung ist nicht zum Nulltarif zu erhalten. Die Kosten der Ener- giestrategie2050 sind im Verhältnis zu deren Nutzen mehr als nur moderat. Und davon profitieren kann erst noch primär die schweizerische Wirtschaft. Vielfältige Ansatzpunkte - vom Energiesparen im Gebäudebereich und im Verkehr über den Ausbau der erneuerbaren Energiequellen bis hin zum Atomausstieg werden weiterführen. Bereits jetzt sind intelligente technische Lösungen im Solar- und Windbereich mit den bisherigen Energien konkurrenzfähig. Diesen Entwicklungen gilt es nun - im Sinne von Rudolf Virchow - eine Chance zu geben, denn sie sind Voraussetzung für unsere Gesundheit. Mit einem Ja zur Energiestrategie am 21. Mai 2017 geben wir uns selber diese Chance - und noch wichtiger: unseren Kindern und Kindeskindern!

Literatur

1 Virchow R. Abhandlungen zur wissenschaftlichen Medicin, Frankfurt, 1856, zitiert in 2. unveränderter Auflage, 1862, S. 56.

2 Virchow R. Die Epidemien von 1848, in: Virchows Archiv 3, 1. u. 2. Heft, 1851, S. 7.

3 Virchow R. Über die Heilkräfte des Organismus. Vortrag, gehalten am 2. Januar 1875 im Verein für Kunst und Wissenschaft zu Hamburg. Berlin 1875, S. 15.

4 WHO - Climate Change and Health, 2016: http://www.who.int/ mediacentre/factsheets/fs266/en/

5 ÄrztInnen-Komitee EnergiestrategieJA: http://www.aefu.ch/ index.php?id=10831

Bildnachweis

Wikipedia:

https://commons.wikimedia.org/wiki/File\%3ARudolf_Virchow.jpg 